\title{
Échanges épistolaires et préceptorat des Lumières
}

André Bandelier

\section{(2) OpenEdition}

\section{Journals}

\section{Édition électronique}

URL : https://journals.openedition.org/dhfles/2871

DOI : $10.4000 /$ dhfles. 2871

ISSN : 2221-4038

\section{Éditeur}

Société Internationale pour l'Histoire du Français Langue Étrangère ou Seconde

\section{Édition imprimée}

Date de publication : 1 décembre 2002

Pagination : 145-173

ISSN : 0992-7654

\section{Référence électronique}

André Bandelier, «Échanges épistolaires et préceptorat des Lumières », Documents pour l'histoire du français langue étrangère ou seconde [En ligne], 29 | 2002, mis en ligne le 01 janvier 2013, consulté le 27 mai 2021. URL : http://journals.openedition.org/dhfles/2871 ; DOI : https://doi.org/10.4000/dhfles. 2871

Ce document a été généré automatiquement le 27 mai 2021. 


\title{
Échanges épistolaires et préceptorat des Lumières
}

\author{
André Bandelier
}

\section{Présentation}

1 L'étude des correspondants suisses du secrétaire de l'Académie de Berlin conduit au fonctionnement de la République des lettres et au rôle assuré par l'Helvetia mediatrix, à l'histoire culturelle thématisée par l'encyclopédisme et les rapports de la science et de la religion ${ }^{1}$. Elle permet aussi de revisiter le thème du préceptorat ancien dans un espace européen qui use du français « langue internationale ».

2 La correspondance essentiellement passive de Jean-Henri-Samuel Formey (1711-1797), secrétaire «éternel » de l'Académie royale prussienne de 1748 à sa mort, comprend plus de 17.000 lettres pour plus de 2.400 épistoliers. L'usage des langues s'y révèle très inégal : $90 \%$ des lettres sont en français, le reste se partage entre le latin et l'allemand. La disproportion est plus marquée encore parmi les 180 Suisses et leurs 2.500 missives : un théologien zurichois recourt au latin; les lettres en allemand se comptent sur les doigts de la main; même Albrecht von Haller, une des gloires de la littérature germanique, s'exprime dans la langue de Voltaire.

Romands et Alémaniques mêlés par l'usage élitaire du français écrivent des cantons suisses et de leurs "pays alliés " dans un tiers des cas, mais surtout de l'étranger. L'éventail va de Charlestown en Caroline du Sud à Saint-Pétersbourg, en passant par Londres, Copenhague, Stockholm, Riga. Ils se concentrent dans les Allemagnes, en Prusse et en Saxe notamment. Ils forment un triangle culturel extrêmement fécond, d'inspiration huguenote, dont les sommets pourraient être Amsterdam, Genève et Leipzig. Berlin, Berolinum, anagramme lumen orbi(s), allemand Licht der Welt, offre le pôle fédérateur en la personne de Samuel Formey, fils de réfugié français né dans cette ville, prédicateur, journaliste polygraphe, traducteur, intermédiaire culturel idéal dans la tradition de la République des lettres². 
Henri Duranton avait déjà utilisé cette volumineuse correspondance en 1985 pour «Précepteurs, demoiselles de compagnie et bohême littéraire dans le Refuge allemand ». Resté prisonnier de sa source, il avait dressé un tableau misérabiliste de ce "prolétariat intellectuel », résumé par un quatrain sans date de Jean Amira :

Etre à Berlin

C'est un métier de chien

Et je voudrais bien être à la porte.

Mais le Diable ne veut pas que j'en sorte ${ }^{3}$.

Dans l'intervalle, Franz Rudof Weller, Konrad Schröder et Herbert Christ ont présenté les pays allemands au XVIII ${ }^{e}$ siècle comme un remarquable terreau d'innovation, tant au niveau de la didactique des langues qu'à celui de l'éducation en général ${ }^{4}$. Deux éléments avaient retenu mon attention dans l'article de Duranton: la présence de Suisses parmi les épistoliers; le choix des extraits qui laissaient augurer, conviction loin d'être confirmée à l'analyse, que le corpus contenait ces à peu près des pratiques enseignantes que je recherchais ${ }^{5}$.

\section{Précepteurs et gouvernantes " suisses ${ }^{6}$}

6 Parmi les correspondants suisses de Samuel Formey, nous comptons au moins une trentaine de précepteurs et gouvernantes (voir Annexe in fine). A travers eux, nous touchons à des figures centrales de la société d'Ancien Régime : intermédiaires entre les générations, les ordres et les cultures ${ }^{7}$. Par l'usage dominant du français, ils représentent aussi des personnages clés de la didactique des langues vivantes, comme en témoignent les usages lexicaux du temps. En pays germanique, on reproche ainsi au continuateur de Leibnitz à Halle le philosophe Christian Wolff de se prendre pour «le précepteur du genre humain $»^{8}$. Et la version simplifiée du Meidinger, Erste Unterricht der französichen Sprache für Kinder, traduit encore en 1794 l'allemand « der Lehrer » par «le précepteur».

Une statistique élémentaire permet de retoucher d'emblée l'image de ces intermédiaires culturels. Grâce à des recherches complémentaires, la carrière de 25 hommes peut être reconstituée. Ils ne vous sont pas tous inconnus: Jean-Jacques Rousseau, Jean-Paul Marat... A cette aune, les touches sombres du tableau de Duranton font place à davantage de potentialités, à plus de diversité dans les devenirs. La destinée des 5 femmes, dont la formation dut être essentiellement familiale, reste plus difficile, voire impossible à cerner. L'ascension sociale des 12 anciens étudiants en théologie, 5 en philologie et belles-lettres, 4 en droit, 2 en médecine et 2 autodidactes, reste impressionnante, même si le préceptorat entraîne quelques Suisses dans la misère. Ils forment un contrepoids important à la présence française à l'Académie des sciences de Berlin avec 10 membres, dont 3 remplirent la fonction de directeurs de classe et 1 celle de secrétaire perpétuel ${ }^{9}$. Parmi eux, nous enregistrons 4 anoblissements et repérons les lectrices de la reine-mère de Suède et de la margrave von Schwedt. Leur champ d'action géographique s'oriente résolument vers le Nord et l'Est: forte assise dans les principautés allemandes et présence dans l'Europe nordique et orientale. Le tiers exercent leurs talents en Prusse; 2 ou 3 dans chacun des Etats suivants: Provinces-Unies, Danemark, Suède, Pologne, Russie. Sans oublier la Saxe et la Lusace, la Poméranie, la Hesse et le Palatinat, Hambourg, mais également la France des familles protestantes, Londres. 
8 En fait, la relation épistolaire peut être décevante pour le sujet qui nous occupe, même si le secrétaire perpétuel de l'Académie de Berlin tient une place centrale dans le réseau des précepteurs et gouvernantes. D’une part, les échanges avec Berlin ne sont pas forcément contemporains de l'exercice du préceptorat. D'autre part, ceux qui l'exercent n'orientent pas nécessairement cette correspondance sur leur profession. La structuration de l'ensemble exploitable, c'est Herbert Christ qui l'offre en distinguant «sept modes d'accès au français ${ }^{10}$. En effet, le corpus conduit à trois d'entre eux : l'apprentissage précoce par les bonnes et les gouvernantes; l'enseignement privé aux enfants et aux adolescents; l'enseignement dans les académies de nobles. Le peu de signification des glanures relatives à l'Académie royale des gentilshommes à Berlin et au Corps des cadets de Saint-Pétersbourg ${ }^{11}$, permet de réunir le tout sous le label de l'enseignement privé. Mais la diversité des situations engage à distinguer les conditions du gouvernorat féminin, du préceptorat domestique et du gouvernorat princier.

\section{Le gouvernorat féminin}

9 La correspondance entretenue avec Berlin par les gouvernantes introduit d'abord à des réseaux d'embauche. Elle conduit ensuite à une condition sociale davantage qu'à des pratiques enseignantes. Ces femmes, conscientes de leur fonction et de leur éducation, parfois de leur naissance, éprouvent un besoin très fort de reconnaissance. Ne voulant passer ni pour servantes, ni pour maîtresses d'école, encore moins pour simples maitresses de français, elles affirment la primauté de l'éducation sur l'instruction dans la tradition de la gouvernante vouée à former le cœur de sa ou de ses pupilles et à inculquer le respect du divin.

Elles assurent souvent le recrutement de consœurs, en particulier quand l'expérience ou l'éloignement en font des informatrices très sollicitées. Il en est ainsi de SusanneElisabeth Lequin, d'extraction noble, que le décès d'un mari contraint à assurer à l'étranger l'avenir financier de ses enfants restés au pays. De Riga dans l'ancienne Livonie, elle facilite par exemple en 1768 l'engagement d'une gouvernante et par là réunit pour nous les qualités recherchées pour cette fonction, présentée ici exceptionnellement comme un état valorisant :

si comme vous le faite écrire a Mademoiselle Molinie cette Demoiselle [de Ketler] a un mérite solide quelle parle un fransai épuré quelle soit douce et amème de former le Coeur dun Anfans, je suis chargée de la part de Monsieur le Baron de Budberg de lui marquer quil accepte ces services avec bien du plaisir. (...) Cest réellement une plasse qui convient a une personne de condition ${ }^{12}$.

11 Les relais du réseau éducatif sont également ménagés par des gouvernantes proches $d u$ pouvoir, ou qui l'ont été, telle Henriette Müller, ancienne lectrice de la reine-mère de Suède, active dans de grandes familles russes, ou encore une Française associée aux correspondants helvétiques et lectrice d'une princesse allemande, madame de Prémontval ${ }^{13}$. Deux amies placées simultanément à Torgau au début des années 1760 donnent des illustrations plus complètes de leur condition mais restent muettes sur l'enseignement du français.

12 Salomé Monod, qui sollicite les directions du prédicateur Formey sur ses projets de mariage avec un Neuchâtelois commis au fourrage et aux provisions de l'armée, se présente elle-même : 
Je suis une fille de 25 ans qui quittai Berne en Suisse il y a trois ans et demis, pour venir en Saxe joindre une Tante qui y est depuis plusieurs années. Elle me plaça ici [à Torgau] chez Monsieur le Chambelan de Preuss en qualité de Gouvernante pour sa jeune famille. [...] il a plut a Dieu a me faire naitre dans une condition destituée de tous les biens de ce monde : Mes chers Parends ont tachés de supleer a ce defaut, en me donnant une bonne education.

Sa vocation reste tout entière dominée par des considérations religieuses et la crainte qu'un séjour à l'étranger puisse affaiblir ses convictions réformées :

Je n'ai épargné ni soins ni peine pour repondre dignement a la vocation dont Dieu ma revetue. J'ai taché de rendre mes élêves bonnes et vertueuses en leurs aprenant a conoitre l'Auteur de leurs Etre et de tous leurs biens ensorte que je crois m'avoir acquis la bienveuillance de Dieu et celle de mes Maittres. [...] ce qui me degoute le plus de la Saxe est que sachant tres peu d'Alman je suis privée, de tout exercices public de pieté, et ne puis communier que toutes les Année une fois.

Mal secondée par des parents trop complaisants pour leur nombreuse progéniture, elle ne peut quitter la Saxe luthérienne pour Vienne la catholique, en dépit d'une charge exténuante et de l'attrait d'un gain supérieur :

il est vrai qu'au premier coup d'oeuil je jouis d'un sort suportable, il le seroit en effet si j'avois moins que sept Enfans sous ma direction, qui sont nés d'une Mère indulgente, qui ne leurs done aucune dicipline, ni aucune authorité à ceux qui sont chargés de leurs éducations Coment donc s'y prendre pour élever ces jeûnes plantes avec toutes les qualites requise dans de veritable Chrétiens? C'est pourtant un conte que je dois rendre à Dieu ? [...] Il y eut ici un Comte de Vienne, qui m'a offert cent Ducats si je voulois aller élever une fille unique qu'il avoit. Je n'aurois pas manqué d'accepter cette occasion si la Religion ne sy fût opposée et ne m'eu fait envisager tous les perils auquel j'auroit éte m'exposer en m'en allant dans un pays d'une Religion si contraire à la mienne ${ }^{14}$.

Salomé Monod n'est pas la seule à prendre pour patron l'auteur du Philosophe chrétien. Ainsi celle qui se nomme elle-même «la Deyverdun" s'inspire de la femme sage du Philosophe chrétien, ou Discours moraux et la donne pour modèle à ses élèves. Cet ouvrage clé de Formey a été refusé à Paris, mais édité à Leyde chez Elie Luzac de 1752 à $1757^{15}$. Le philosophe y développe les qualités habituellement réservées à la femme, mais non sans remarquer qu'elles seraient "bienséantes aux deux sexes" et non sans s'interroger sur « la distribution juste ou injuste, (c'est un problème indécis), qui s'est faite des occupations de la Société $»^{16}$.

Louise Deyverdun et ses tribulations permettent de compléter le tableau : aptitudes et conscience de soi, conditions de travail, rapports avec ses employeurs, développement d'un réseau d'assistance ${ }^{17}$. La correspondance se noue en 1760 autour du besoin de reconnaissance, après quatre années passées à Torgau chez le général de Plötz. La Suissesse attend de Formey davantage qu'une simple « condition » à Berlin pour celle qui se revendique d' ' une certaine naissance et education » : « je ne regarde point a de Gros apointemens, mais aux bonne manieres d'une Dames desprit qui sçait Mettre quelque differance entre une Gouvernante et une Servante ». La lettre suivante sera l'occasion d'un portrait féroce de Madame, "une personne de petite Extraction", soupçonneuse et colérique. Une première négociation pour le comte de Rexe échoue suite à l'intervention probable de ses maitres. Elle a pu observer alors que les promesses d'un seigneur de cour ne sont trop souvent que « du vent»; sa consolation restera d'avoir su inspirer "des sentimens chretiens » à ses élèves. C'est pourtant l'occasion d'un commentaire sur cet arrangement non honoré et d'une découverte des aptitudes et vœux de la postulante qui refuse les travaux réservés au sexe faible : 
à lappointement je nay rien adire, ce ne sera jamais a quoi je regarderai il faut ce reserver les frais des voyages pour aller et le retour au cas qu'on ne sacomode pas 2, le caffe en nature ou En argens quand aux Etrenes je m'en remet a leur génerositez, plus je ne m'engage point a faire des ouvrages come bien des dames le pretande en Saxe [...] plus je ne possede pas la langue allemande n'en sachant que ce quil faut pour demander ce qui met nécesaire. je crois que ce nest pas un deffaut pour une Gouvernante ${ }^{18}$.

17 Ayant accompli ses trois mois de dédite et après un crochet par Leipzig pour communier dans la confession réformée, elle s'installe fin 1761, encore en Saxe, chez le chambellan de Schönberg à Thamenhayn près de Würtzen. Dans l'intervalle, elle a sagement renoncé à une sollicitation pour Dresde qui dépassait ses compétences : « jai quelque connoissances de ces siences, mais pas assez pour les Enseignier afond, par consaiquand je ne puis acepter une condition ou on les pretens pour n'en pa sortir avec honte ». Elle juge alors son sort supportable: 100 écus de gages, le café, certaines attentions comme de pouvoir disposer des chevaux pour aller visiter le voisinage ; une élève de douze ans, fort douce, dont elle est la première gouvernante, et une brave maîtresse ; des loisirs consacrés à la lecture plutôt qu'à l'ouvrage. Le hic, c'est Monsieur dont on parvient tout de même à contourner l'avarice :

lors que je veut quelque chose pour mon Eleve, je lui dis nous aurion besoins de telle chose mais cela est trop couteu il ne dit mot quelques jours après je lai [...] ceux qui vivent avec de tel jens ne peuvent Etre heureu qu'en Etudians leur foibles et les prendre par la ${ }^{19}$.

On la retrouve ensuite à Nassenhaide en Poméranie, d'où elle avoue bientôt à Formey ne plus pouvoir supporter davantage « les brusqueries de Mad la Comtesse » de Lepel. Le projet de fonder une pension à Berlin avec son amie Villard-Monod, devenue veuve prématurément en 1763 , ne semble pas s'être concrétisé. Ses pérégrinations la mènent à Gollnau chez le lieutenant-colonel de Froideville, puis en 1764 à Grauvienckel chez Monsieur de Globig. Après avoir cru être passée de l'enfer au paradis, elle y déchante en découvrant la duplicité de ses supérieurs :

\begin{abstract}
je vais vous instruire en deux mot d'un trait qu'on me prepare que jai apris par une lettre qui met tombe par hazard entre les main elle est adressée a Mr de Globig elle est d'un vieux maitre de langue françoi en date du moy passez. je me rejouit daprendre que vos enfans savent deja lire et entendre le francoi du moins en partie. sil continue a faire des progres vous avez lieu de croire quil naurons plus besoin de demoiselle a pâque, jacepte sans Esiter loffre que vous me faite de venir prendre sa place ; afin denseigner les regles de la langue latine [et] françoise a Mr vôtre fils pour Epargner un Gouverneur pandant quelques année [alinéa] ainsi je serois venue yci pour faire la maitresse decole et me faire donner mon Congé.
\end{abstract}

19 A la duplicité « colorée de toutes sorte de bonne façon [et] promesse », elle répond une fois encore par la dignité : « javoue quil ma falu vincre pour ne rien faire paroitre, mais je suis resolue de a me pour voir allieur dela premiere Condition sortable qui se presentera $»^{20}$.

Ses déboires ne l'empêchent pas de s'entremettre pour d'autres, parents ou connaissances qui, chacun à sa manière, entendent profiter de la vogue du français en Allemagne. Elle présente à Formey les difficultés de Jacques-Georges Deyverdun, gouverneur en Haute-Lusace, et favorise par là l'entrée de son cousin germain au service des Wurtemberg ${ }^{21}$. Puis elle intercède pour $\mathrm{M}$. Zollner, gouverneur du jeune comte de Lepel. Mais quand Formey propose une place dans un collège à celui qui " parle Anglois, italiens, françoi fort joliment» outre les « la[n]gues necessaires à sa 
vocation » de théologien, la gouvernante précise que ce collègue vise plutôt le SaintMinistère. On retrouve alors la vénalité d'une époque où tout état, fût-il ecclésiastique, est susceptible d'être négocié : «lami vient me dire que sil etoit possible davoir une paroisse suffisement lucrative pour y vivre honêtement il fera un present de cent Ecu que je mettrai en depos chez vous a mon arrivée a Berlin et pour Aumonier [dans un régiment] 50 tout argent de or au debour ". Dans le même temps, la Deyverdun s'emploie à secourir à Berlin la veuve Villard et ses parents. La seule issue lui semble encore venir de l'enseignement : «le pere possede lAritmetique et Ecrit tres bien, je ne vois dautre resource pour Eux que de com[men]ser une Ecole, aidé par votre Généreuse protection $»^{22}$. Enfin elle joue les intermédiaires entre la sœur de Madame de Globig, qui offre 60 écus pour s'occuper de sa fille de 11 ans, et une protégée du secrétaire de l'Académie des sciences. Mais elle s'emporte quand une lettre de l'intéressée juge, sur des ouï-dire, la postulante trop capricieuse et trop âgée : «je lui ay repondu qu'une autre fois au lieu de sadresser a moi elle devoit sen faire peindre une suivent son gout ». Et conclut le chapitre des employeurs de manière péremptoire: «nos Dames saxone deviene toujour plus fentasque surtout les Campagnardes pour la miene vient de m'en donner un trait de sa, comment diraije bétise ou sotise, non mais malice noire $»^{23}$. Sans préciser la raison de son courroux.

\section{Le préceptorat domestique}

21 Gouverneurs et précepteurs en relation épistolaire avec Formey intègrent leur statut d'enseignant à la course générale à la fonction et à la rente. Ceux qui œuvrent dans un cadre familial étendent la couverture spatiale aux Provinces-Unies et à la Russie et offrent d'autres champs à la langue française hors de France. Mais je n'ai pas retrouvé les relations précises exhumées de la correspondance d'un Théophile Rémy Frêne. Rappelons que celle-ci permet de présenter le précepteur francophone en Allemagne à la veille de la Révolution comme un maître généraliste qui ne s'exprime qu'en français ${ }^{24}$ et qui tire généralement profit d'ouvrages moins spécialisés que les manuels existants pour ses leçons de langue. Quant au Suisse maître de français à Londres ou dans les petites académies provinciales du sud de l'Angleterre pendant la Révolution, il doit, pour survivre, assumer la fonction plus large de maitre de langues: enseigner l'allemand, l'italien voire l'anglais pour les leçons privées au domicile des élèves, au gré de la concurrence évolutive entre les langues ; ou dispenser les humanités gréco-latines dans les académies. La vocation du préceptorat semble souvent absente et quand elle est bien réelle, elle ne justifie que rarement l'entretien de la correspondance, excepté le cas où on recourt à l'efficace réseau du secrétaire de l'Académie de Berlin pour une première place ou un changement de situation.

Aussi les lettres envoyées par le Neuchâtelois Jean-Laurent Garcin ne portent-elles pas d'abord sur ses activités de gouverneur aux Provinces-Unies, mais sur sa participation à la République des lettres et sa collaboration avec Formey journaliste et auteur publié en Hollande ${ }^{25}$. C'est incidemment qu'on le voit passer de la condition de gouverneur chez le secrétaire de la ville d'Amsterdam Abraham Calkoen à celui d'accompagnant de deux jeunes Amsteldamois, les frères Gerard et Willem Munter, étudiants en droit à l'Université de Leyde ${ }^{26}$. De cet état n'émerge que le souhait plus profond d'occuper simultanément une vocation de théologien et une chaire d'éloquence ou de belleslettres françaises. Mais son renoncement à Copenhague incite à se méfier des 
extrapolations à partir d'apparences somptueuses. Au reproche d'inconstance formulé en 1763 par son correspondant de Berlin, Garcin rétorque en se fondant sur l'appréciation de son ami Reverdil : «Il m'a répondu que cette Chaire [de belles-lettres françaises] était tombé depuis M. Mallet, et que de son tems meme, l'Auditoire du Professeur était désert $"^{27}$. En réalité, les relations de confrères gazetiers et polygraphes nourrissent l'échange épistolaire. Garcin cherche à intéresser Formey à la diffusion de son anthologie de psaumes en vers français. Formey journaliste et Garcin qui, avec l'exjésuite Robinet, poursuit en Hollande l'impression d'un périodique qui a cessé de paraître en France, le Journal étranger ${ }^{28}$, partagent le même intérêt à s'échanger des nouvelles littéraires. Le gouverneur, intermédiaire culturel, pose la question qui nous fait regretter de ne pas posséder la réponse du Berlinois : doit-il instruire des livres qui paraissent en hollandais ou de ceux qui s'impriment en Hollande? Surtout, en 1763-1764, le Suisse collabore à l'impression d'ouvrages de Formey à Leyde, chez Luchtmanns et Luzac, plus directement à la parution de l'Emile chrétien chez Jean Néaulme à Amsterdam. Ce n'est pas sans signification; les compilations qui mirent en fureur Jean Jacques font de Formey, opposant à la pensée de Rousseau, le paradoxal diffuseur de ses idées en Allemagne ${ }^{29}$.

Dans le même temps, un parent assez éloigné, le pasteur Jacques Garcin, occupe le poste de "professeur ordinaire de langue française " [gewoon hoogleraar Frans] à l'université frisonne de Franeker depuis avril 1755. Sa leçon inaugurale s'intitule comme de juste De utilitate linguae Gallicae. Mais le qualificatif de "maître de langue " [taalmeester] et un salaire de 350 florins par an, plus de cinq fois inférieur à celui d'un collègue de la Faculté de droit, n'indiquent guère que sa chaire ait été prise très au sérieux ${ }^{30}$. L'éloge controversé d'un collègue théologien adepte du wolffianisme le rapproche de Formey en 1763. Il nous vaut un rappel de la carrière de Garcin. Avant d'exercer le Saint-Ministère aux Provinces-Unies, il a tâté une décennie durant du gouvernorat, d'abord dans trois grandes maisons de Berlin et de Francfort sur l'Oder, chez MM. de Masson, Wartensleben et Schmettau, puis comme précepteur chez un négociant de Hambourg. Cette évocation confirme l'objectif prioritaire des gouverneurs et précepteurs, à savoir l'aspiration à une situation stable qui viendrait récompenser leur véritable «noviciat» d'enseignant et d'éducateur, et le meilleur accueil généralement reçu dans la bourgeoisie commerçante. Le portrait que Garcin dresse de la fonction n'est pas sans anticiper non plus le professeur du XIX siècle, le " prolétaire en jaquette » d'un Jules Vallès :

Je n'aurois jamais quitté le Brandebourg, si j'y avois été plus heureux, \& si les Pères de mes Elèves dont à l'exception du prémier je n'ai eu que peu ou point de sujet de contentement s'êtoient interessés à m'y procurer quelque établissement solide selon leurs promesses, \& mon application à remplir fidelement tous les devoirs de mon Poste d'alors, mais après avoir vécu chès Eux dans une brillante misére \& avoir $\mathrm{vu}$ pendant près de sept ans le peu de fond qu'il falloit faire sur leurs paroles emmiellées je me décidai à en sortir ${ }^{31}$.

24 Arrivé à Moscou en septembre 1784, le Vaudois Fornerod attend de Formey, correspondant stipendié par l'Académie de Saint-Pétersbourg, des protections afin d'échapper à sa condition de précepteur, exercé notamment en Russie blanche ${ }^{32}$. Installé dans la même fonction chez le général prince de Galitzine après un essai infructueux à Saint-Pétersbourg, il vise " une bonne occasion pour voiager ", sinon la place de professeur en langue française et belles-lettres à l'Université de Moscou que le titulaire semble bien mal illustrer : 
Celui qui l'occupe aujourd'hui, est si occupé de son commerce de confitures, marinades, huile d'olive \&c, de pretter sur gages, \& de faire des affaires, quil néglige entièrement ses leçons, \& qu'on s'attend d'un Jour à l'autre à le voir congedié.

En attendant mieux, il partage la situation du préceptorat domestique peu instruit de « ces climats hyperboréens »:

Des Places de Precepteur, je n'en manquerai pas, elles sont si communes, \& l'on est si peu délicat, quil n'est pas de Laquais, tailleur, cordonnier, charcuitier, deserteur \&c, qui ne soit sur d'en trouver une, pourvû qu'il parle Français, \& qui ne puisse y recevoir 4, 5, \& $600 \mathrm{R}$ [oubles] d'apointemens.

Sa condition est aggravée par la pingrerie d'un riche employeur :

[Le prince de Galitzine] est d'une avarice incroiable, n'ayant qu'un fils, \& trois filles, il est si peu délicat sur leur éducation, qu'il a donné successivement pour gouverneur à son fils, un garçon parfumeur, \& un valet de chambre perruquier, son dernier que j'ai remplacé à un frere Laquais dans une autre maison. Il a pris de tels miserables parce quil les avait à bon marché.

De plus, le comportement du fils l'incite à quitter le service pour ne pas devoir répondre de ses « infamies " prévisibles : «mon Eleve agé de onze ans, beau comme un ange, \& plein d'esprit, a le coeur si furieusement depravé, par les instructions \& les mauvais éxemples des Précepteurs quil a eu avant moi, que j'en suis éffraïé \& revolté $\aleph^{33}$. Les recommandations parviennent bientôt de Berlin, ainsi que les commissions ; loin de changer quelque chose à la condition du gouverneur, appointé à 500 roubles par an sans perspective ni d'augmentation ni de voyages, elles obligent à $d$ 'humiliantes démarches. Après avoir été éconduit plusieurs fois, Fornerod obtient une entrevue d'un quart d'heure avec le comte Bruce. A l'énoncé des souhaits, «quelque place de secretaire, Bibliothecaire, homme de compagnie, \&c chès un grand Seigneur, ou quelque occasion pour voiager avec un Jeune homme de famille», celui-ci lui rétorque "quil avait crû qu'il ne s'agissait que d'une place pour enseigner de jeunes Enfans».

L'entretien de deux heures avec le général Melissinow, curateur de l'Université, mérite un large écho, ne serait-ce que pour la tentative de contrôle de l'enseignement domestique par cette institution:

En lui présentant votre Lettre: Est ce que Monsieur le Conseiller privé Formey se souvient encor de moi ? Après l'avoir lue, il m'assura en termes très obligeans, qu'il s'emprèsserait à faire honneur à la recommandation d'un homme quil éstimait \& réspectait éxtrèmement. Et pour m'en donner à l'instant une preuve, il me conseilla très sérieusement de commencer par me conformer aux ordres de sa Majesté, qui portaient, que tout Etranger qui voulait se charger ici de l'emploi de Précepteur, devait se faire éxaminer à l'Université, \& en prendre un certificat sous peine à tout contrevenant, d'etre transporté hors des frontières - Revenu de la surprise ou ce conseil serieux \& amical m'avait jetté, Je repondis en souriant, que depuis six ans que j'avais le malheur d'éxercer le désagréable metier de Précepteur en Russie, personne n'avait jamais douté de mes petits talens, \& qu'on ne m'en avait jamais demandé un certificat, en ayant tant d'autres que je pouvais présenter; Que mon petit amour propre repugnait beaucoup, à me faire éxaminer par un Professeur Baudet, ou Baudoin, qui de notoriété publique, passait pour bien mieux se connaître en anchois, huiles d'olives, \& saucissons de Boulogne qu'en Littérature Française; Qu'enfin je ne croiais pas un tel certificat nécéssaire, puisque je ne desirais rien avec plus d'ardeur, que de trouver l'occasion de quitter un état pour lequel je n'etais pas fait, \& qui me déplait chaque Jour davantage - Il me repondit en riant, qu'il n'avoit point pensé qu'un homme comme moi serait éxaminé sur la sellette, par les Professeurs de l'Université comme on le fait aux misérables avanturiers 
Français qui s'y présentent, que tout cela n'eut été qu'une affaire de forme, pour avoir un certificat, mais quil ne m'en parlerait plus, puisque j'y montrais de la repugnance, \& que je pourais à la rigueur me passer de certificat. J'en pensais de même, puisque jamais personne ne m'en a fait aucune mention, \& que la moitié des misérables Précepteurs qu'on voit ici, n'osent pas, ou ne veulent pas en demander, \& qu'ils passent également. honorés de recevoir un billet du secrétaire de l'Académie prussienne mais qui répugnent à répondre à un intermédiaire non titré : aussi bien la recommandation d'une gouvernante que le projet d'une correspondance entre l'Académie de Berlin et l'Université de Moscou achoppent à cet écueil.

Au bénéfice de recommandations genevoises efficaces, Paul-Henri Mallet ${ }^{34}$ marchande les conditions de son contrat avant d'accepter le service du comte de Calemberg, « conseiller privé en Saxe » installé en Haute-Lusace. Cette place à moins de 500 livres par an, frais de voyage en sus, il la doit à Formey qu'il a sollicité doublement en février 1751 : la nécessité le pousse à chercher «quelque sorte d'etablissement hors de [sa] patrie »; il souhaite asseoir sa réputation par quelques pièces en vers, dont une Epître au roi de Prusse, dans la Bibliothèque germanique. De fait, si Mallet livre la description la plus décoiffante de l'environnement d'un précepteur au milieu du XVIII ${ }^{e}$ siècle, sa soif de reconnaissance balise le passage du préceptorat privé au préceptorat princier.

J'ai trouvé, suivant qu'on me l'avoit représenté, un Seigneur, et une Dame polis, raisonables, éclairés. Dans le Seigneur de l'affabilité, des lumières, du goût, du quant à soi. Dans la Dame un abord froid, un entretien sensé, des égards, et de ce qu'on appelle vivacité chez les gens de Condition. Pour mon elève, je ne m'etois point fait de peinture anticipée de son caractère, et j'ai bien fait, car c'eût eté en pure perte. C'est un vrai lutin, un Diablotin incarné, beau comme l'amour, plus malin encore, et aussi incapable de laisser quelque repos à ceux avec qui il a affaire. Depuis que je le connois, j'ai ajouté à mes prières l'article du don de la patience ; j'en repete le voeu 15 ou 20 fois par jour, mais je vois bien qu'enfin je serai obligé d'en rompre la tête à tous les Saints, jusqu'à ce que je ne sache plus auquel me vouer. Il y a déja eu entre lui et moi, dans le cours de cette semaine 50 ou 60 demelés et autant de reconciliations. il se ruine en promesses, comme moi en menaces, j'ai affaire à une ecole entière, il se reproduit dans tous les coins de la chambre, et mon oeil ne peut suivre toutes ses etourderies, bien loin que mes reprimandes les atteignent. heureusement il $\mathrm{y}$ a ici une très belle bibliothèque, et j'ai environ trois heures de loisir par jour pour en profiter, et effacer par les charmes de quelques études furtives, toutes les impressions d'ennui, ou de dégout qui auroient pu me rester. Ajoutez a cela quil me paroit que notre voyage de Dresden prend l'air de se réaliser. que là j'aurai plus de loisir, mon eleve ayant plus de maitres, que j'y verrai des visages francois, gibier fort rare ici ou a la reserve du Seigneur et de la Dame, tout le monde parle allemand, ou ecorche le francois, come mon éleve par exemple qui tantôt ne voulant pas m'entendre, et tantôt ne le pouvant pas, se trouve par le fait sourd avec moi, et me rend muet avec lui ${ }^{35}$.

Le voyage de Dresde toujours remis à plus tard, Mallet aspire à échapper à sa «solitude ennuieuse » : « jappelle solitude, un séjour ou l'on ne voit rien, et ou je n'entens que de l'allemand hors le tems que je passe avec mon elève. La conversation qu'on a ici pendant le diné etant très silentieuse, et la seule pourtant que j'aye avec leurs Excellences ${ }^{36}$.

33 Il se rend compte que sa soif de reconnaissance implique la recherche de protections et faveurs que la Haute-Lusace ne peut lui offrir: «Des caresses, des manieres affables, 
des lettres seduisantes tout cela ne coute pas grand chose a des gens nourris de la culture des plus polis auteurs de [F]rance ». Surtout, Mallet qui a déjà couvé un dessein semblable avec Marseille (liaisons non abouties), veut briguer le titre de correspondant de quelque académie provinciale française et le soumet à Formey. La légitimation passe par la situation du vécu saxon:

faute d'etre conoisseurs on juge uniquement des gens de lettres par ce qui leur est le plus etrangers, des titres, des distinctions etc. Il faut être instalé home de mérite par quelque chose d'autentique sans quoi on vous conteste vos titre, et un home de lettres est regardé comme un maitre de langue, un home obscur et mercénaire ou un Pedant.

Un tableau peu flatteur des académies provinciales sert de réfutation anticipée à qui s'interrogerait sur les mérites du jeune homme : « vous savez sur quel pié sont quelques académies de [F]rance. Confinées dans la province, et souvent reduites a choisir ce qui se trouve plus que ce qu'elles venêroient, elles ont eté forcées a ne pas aspirer a avoir des associés bien distingués, et a ne pas faire les difficiles ${ }^{37}$. Formey consent et écrit en particulier à Voltaire, qui a reçu antérieurement des vers de Mallet ${ }^{38}$. Mais son entrée à la Cour de Danemark en 1752 se passe sous d'autres auspices.

\section{Le gouvernorat princier}

On a souligné la forte présence de francophones dans l'entourage des princes allemands pour leur éducation. A cet égard, la correspondance de Formey garde des traces significatives de protestants suisses dans les Cours d'Allemagne et du Nord. En faisant appel à ceux-ci voulait-on aussi éviter la prétendue frivolité et le supposé laxisme des Français, comme on le sous-entend parfois? En l'occurrence, le secrétaire de l'Académie prussienne constitue un destinataire très privilégié : l'appartenance à la République des lettres et la réception dans une société savante restent des signes d'élection incontournables pour gouverneurs et précepteurs princiers quelle que soit leur origine. Peu de choses filtrent sur la personne des futurs souverains, car la discrétion reste de rigueur en dehors de la Cour. Il n'en résulte guère d'informations supplémentaires relatives à la didactique des langues étrangères. En revanche, le manque de substance au niveau pédagogique laisse plus largement la place à l'intermédiaire culturel. L'image du préceptorat en sort valorisée, en particulier par les échanges qu'il favorise entre les cultures. Plus prosaïquement, le gouverneur ou le précepteur reconnu se trouve dans la position de distribuer à son tour des recommandations appréciées dans la course générale à la fonction et à la rente qui caractérise l'Ancien Régime.

C'est sans doute grâce à son affiliation maçonnique précoce que Paul-Henri Mallet doit sa nomination en 1752 à la chaire de belles-lettres françaises de l'Académie de Copenhague, pour succéder au protestant français La Beaumelle ${ }^{39}$. Il se déclare alors « homme de lettres » à 400 écus de pension, familier des ministres Bernstorff et von Moltke, ami du poète allemand Klopstock, mais isolé dans une terre non francophone :

la sociabilité ne fait pas la principale qualité des Danois, \& le francois etant beaucoup moins commun ici que chez vous, je suis privé du comerce de bien des gens par cette difficulté, de m'entretenir avec eux. Il est vrai que je suis avancé dans le Danois, au point de lire \& de comprendre passablement un livre, mais je desespere de pouvoir le parler jamais ${ }^{40}$.

37 Au printemps 1755, il est choisi pour enseigner le français et les belles-lettres au prince royal, confié par ailleurs au gouverneur $\mathrm{M}$. de Reventlau, au moment où paraît le 
premier volume de son Introduction à l'histoire de Danemark. Même s'il a été égratigné pour son français de Suisse par la critique : «Les Journalistes de Trevoux ne m'ont pas fait de quartier Ils me traitent poliment de francois denaturé dans la patrie des Cimbres, \& me reprochent de n'avoir point le Style de Paris ${ }^{41}$.

Nous apprendrons encore que le prince est un bien aimable enfant, qu'il progresse en français et que son gouverneur est d'un naturel droit et intègre. En 1757, on a commandé à Mallet un abrégé de l'histoire de Danemark pour être enseigné en français au prince, mais la fréquentation de la Cour mène au cynisme: "il arrive bien des revolutions en peu de tems dans le pays de la Cour, \& je ne compte plus sur rien, ce qui me coute d'autant moins que je deviens fort indifferent sur la plupart des choses qui m'agitoient autrefois $»^{42}$. En fait, une lettre de 1760 affirme que le prince a appris l'abrégé par coeur avec son professeur. Surtout, Mallet a pu consacrer toutes ses matinées à l'étude et compter sur ses amitiés genevoises à Copenhague : d'abord André Roger, secrétaire du premier ministre comte de Bernstorff, rejoint par Claude Philibert, qui ouvre une succursale de la librairie familiale dans la capitale, et par Elie Salomon François Reverdil, engagé pour s'occuper du Mercure danois, puis pour enseigner la géométrie à l'Académie des Beaux-Arts. De ce cercle protégé en haut lieu, isolé en terre luthérienne, soudé par la parenté et vraisemblablement par l'appartenance maçonnique, ressort en une décennie une oeuvre remarquable de médiation: spécialement les traductions des littératures et institutions nordiques de Mallet, dont l'Edda, de nouveaux périodiques francophones avec Le Traducteur, extrait des feuilles anglaises, et les Mémoires sur la littérature du Nord, complément au Mercure danois ${ }^{43}$.

Des circonstances exceptionnelles ramènent à la génération antérieure et plus concrètement à l'éducation princière : la présence d'une lettre de 1735 à un parent dans la correspondance philosophique entre Jean-Pierre de Crousaz et Samuel Formey; l'appel aux compétences de Crousaz pour assurer le suivi de pensionnaires prestigieux dans les établissements des bords du Léman. Sollicité pour s'occuper à Lausanne de l'héritier des comtes de Frise ${ }^{44}$, Crousaz y voit une occasion de poursuivre son oeuvre pédagogique. Convaincu que l'art d'élever la jeunesse est uniquement affaire d'expérience, il s'appuie sur sa pratique comme gouverneur de S.A.S. le prince Frédéric de Hesse et livre un ample complément au Traité de l'éducation des enfants (Lausanne, chez Vaillant et Prevost, 2 t., 1722). Son art d'éduquer les Grands tranche résolument avec les canons de la Cour de France. Là où les études confinent l'apprentissage du pouvoir à préparer le roi à la solitude du pouvoir par la priorité accordée au contrôle et à la régulation des passions ${ }^{45}$, Crousaz insiste sur la conciliation nécessaire du coeur et de l'esprit. Il applique ce principe notamment à la religion : il veut la faire aimer en saisissant les occasions les plus agréables d'en parler; il renonce à la catéchisation, mais sans abandonner les secours de la raison. Il révèle une faculté de pénétration particulièrement aiguë :

Je say qu'il ne faut point avoir pour les jeunes gens d'indignes complaisances; mais je say encore plus qu'on ne bâtit utilement qu'à proportion que l'on gagne le coeur, \& que toute voye de contrainte, ne manque jamais de tourner mal, par rapport aux Grans Seigneurs. Je say encore qu'il faut cultiver la raison \& ne pas se borner à enrichir la mémoire. Il faut adroitement conduire l'esprit de celui qu'on enseigne, en sorte que lui même, mis sur des bonnes routes, \& placé à propos dans de justes points de vue, vienne à découvrir ce qu'on a dessein de lui apprendre, \& par là l'anime \& le retienne d'autant mieux, qu'il le regardera comme sa propre production, son propre ouvrage ${ }^{46}$. 
Lhabituelle énumération des disciplines (latin, religion, morale, histoire et géographie) fait place aux ouvertures des premières Lumières (association du latin et du français, forte présence de la religion et de l'histoire, mais aussi arithmétique, géométrie, fortifications, mécanique, optique).

41 À propos des langues enseignées, Crousaz rappelle qu'au service de Hesse, il était secondé par "un informateur». Le terme, rare, ne se retrouve que dans l'espace allemand comme équivalent de "précepteur». Il a sans doute été importé par les précepteurs suisses romands à partir de l'allemand "Information » signifiant «enseignement». Adaptant la tradition curriculaire «classique ${ }^{47}$, Crousaz recommande l'approche simultanée du latin et de la langue maternelle, et l'a appliquée au français et au latin ${ }^{48}$. Il mêle auteurs antiques et histoire dans un environnement débarrassé de toute référence «scolaire »:

J'ay passé quatre années auprès de S.A.S. Mgr. Le Prince Frederic, sans qu'il se soit apercu que je luy fisse des leçons \& il me tenoit compte de tout le soin que je luy donnois, comme des complaisances d'un ami, qui n'avoit pas moins en vuë de le divertir que de lui être utile. Mais qui est ce qui est Maître de soi à la Cour ; c'est un privilège que les Princes eux memes n'ont pas \& dans cette multitude de personnes qui les obsedent, il s'en trouve de tems en tems qui saisissent des momens favorables pour abuser de leur attention. [alinéa] Malgré tous les obstacles que j'avois rencontré à l'éxecution de mes plans, je suis venu à bout, (après avoir commencé avant l'age de sept ans,) avant mon départ de Cassel, de faire interpreter exactement de Latin en François \& de François en Latin, Maturin Cordier, Erasmus de Civilitate morum, Terence \& la moitié des Decades de Tite Live. Il avoit fait aussi éxactement l'Histoire du peuple Juif, jusques à la venue du Seigneur \& XII Tomes de l'Histoire Romaine de Latour, et tout cela accompagné sans cesse de Reflexions de la manière dont je le conseille dans mon Traité de l'Education \& cela sans l'avoir fatigué par un dictionaire, ni luy avoir fait aprendre par coeur ni Rudimens, ni Grammaire, ni Syntaxe, dont il savoit pourtant toutes les Regles ${ }^{49}$.

Les représentants de la génération suivante ne nous laissent aucun témoignage de leur pratique pédagogique. Leur correspondance avec Berlin complète le tableau relativement à leur ascension sociale, à la vie de cour, à l'usage de la langue française, partie intégrante de l'étiquette nobiliaire.

Ainsi, Nicolas Béguelin reste plus que discret sur la prégnante influence qu'il exerce auprès du futur Frédéric-Guillaume II de Prusse de 1747 à 1764 . Ce mentorat récompensé par l'anoblissement après la mort de Frédéric II, ne laisse pratiquement aucune trace pour l'histoire de l'éducation ${ }^{50}$. Béguelin dans ses échanges épistolaires avec Formey apparaît comme le personnage dont on recherche la recommandation et se manifeste à travers son activité d'académicien. Les Neuchâtelois recourent à lui pour briguer une fonction au service de Prusse ou dans leur patrie. L'Académie royale des sciences de Berlin revit dans ses billets par la préparation de séances, la proposition de candidatures et de sujets de concours, par des mémoires divers et des notations météorologiques ${ }^{51}$.

On sait que Formey, alerté par Louise Deyverdun, a permis à son cousin JacquesGeorges de quitter le service des princes de Holstein à Koswig en 1761 pour l'entourage des Wurtemberg et du margrave de Schwedt, de la maison royale de Prusse. Deyverdun prétend ne pas chercher fortune à travers cette promotion, mais l'occasion de voyager, comme beaucoup. De fait, aggravée par les circonstances de la guerre de Sept ans, sa nouvelle condition se vit sous le signe de la maladie et de la frustration ${ }^{52}$. Engagé comme «informateur» pour Stettin, il a de la peine à prendre sa place parmi les 
éducateurs des nombreux enfants du duc Frédéric Eugène de Wurtemberg et de la nièce du grand Frédéric, Frédérique Sophie Dorothée, fille de Frédéric Guillaume margrave de Brandebourg-Schwedt. Sans doute jalousé du gouverneur baron von Feilitsch, il commence par ne pas trouver logis à la Cour de Schwedt. Les enseignements prodigués au « prince Louis » en qualité de gouverneur ${ }^{53}$ et la fonction annexe de bibliothécaire de la duchesse de Wurtemberg lui apportent momentanément quelques consolations. A celle-ci, il conseille la lecture des écrivains suisses, Haller, Gessner, se trompe sur la «nationalité» de Wieland et dans son zèle patriote demande ingénument à Formey si Klopstock ne serait pas Suisse non plus. Pédagogiquement, les conseils demandés à Berlin ne mènent guère qu'à l'histoire et à son enseignement en français, mais n'est-ce pas là le moyen indirect le plus usuel de perfectionner un idiome couramment parlé en cour : «Ne conaissez vous point Monsieur? quelqu'abregé d'histoire Romaine, mais qui ne fût point par demandes et par réponses, ou chaque histoire fût separée et traittée d'une manière suivie ».

Il est piquant que dans ce réseau largement opposé aux idées de Jean-Jacques Rousseau, on reste attentif aux progrès de celles-ci : « Je m'imagine que les petits Emiles de Mr. de Prémontwal ne seront pas moins originaux que ceux de Jean Jacques, si les originaux n'instruisent pas, ils amusent du moins, et je m'impatiente d'avoir l'honneur de faire leur conaissance ». L'imbroglio des fonctions ne s'arrange pas : « Nous sommes ici tous ensemble ; Il est dificile et scabreux, d'avoir quatre Maitres a contenter, sans Compter encor leurs assistans. J'en aurai bientôt un de moins, Monseigneur le Prince de Würtemberg part dans quelques jours pour les bains ». De son goût pour les lettres vient le salut: l'Angleterre l'accueille en 1766 pour une fructueuse collaboration littéraire avec son ami Gibbon. Mais il a pu constater alors qu'il n'était plus question de songer vivre de l'état de gouverneur et voyageur :

J'aurais bien souhaitté [...] de faire avec quelque Anglais le Voyage de France et d'Italie; Mais cette carrière que je croyais encor abondante est épuisée; Trop lontems les Gouverneurs Suisses et autres ont dit que les Anglais n'éxigeaient autre chose sinon qu'on ramenat leurs jeunes gens sains et saufs chez eux, et trop lontems ils ont agi en Conséquence; les Anglais ont enfin ouverts les yeux; Ils ont balancé ce que les Gouverneurs étrangers leur coutaient, et ce qu'ils raportaient, et d'après ce Calcul trop clair, malheureusement, Ils ont pris le parti de faire voyager leurs jeunes gens seuls ou avec des Compatriotes ${ }^{54}$.

Enfin, à Stockholm dès 1760, Jean-François Beylon, chapelain de la Cour, d'abord lecteur de la reine-mère Louise Ulrike de Prusse sœur de Frédéric II, puis collaborateur du roi Gustave III, constitue l'un des nœuds nordiques du réseau « huguenot $»^{55}$. Avant d'être chargé de missions diplomatiques en France et en Espagne, il engage précepteurs et gouvernantes. En 1777, il recherche à Berlin le pasteur acceptable pour l'Eglise réformée française de la capitale luthérienne. La présence des Grands du Royaume y donne un ultime reflet de l'ascendant social de la langue française. C'est pourquoi Beylon ajoute aux qualités requises de « l'homme de Dieu », dont Formey s'est attaché à donner le modèle dans ses écrits, cette remarque significative :

ce ne seroit pas assés pour nous, si nôtre Pasteur ne reünissoit pas aux qualités essentielles, cette Politesse et cet usage du monde si necessaires dans ce malheureux siecle, pour faire ecouter la verité. D'ailleurs notre Eglise est sous les yeux de la Cour et très frequentée par la premiere Noblesse. On ne feroit aucune impression sur elle si on ne joignoit a la force du raisonnement les agremens $d u$ discours: Il faut une sainte addresse aujourd'hui plus que jamais, pour en gagner quelques uns ${ }^{56}$. 


\section{ANNEXES}

Correspondants « suisses » de Samuel Formey (liste des précepteurs, gouverneurs et gouvernantes):

- Achard, François (1699-1782)

Précepteur, dans les années 1730 gouverneur de Karl Wilhelm de Finckenstein futur ministre de Frédéric II, membre de l'Académie de Berlin (1744), diplomate et mathématicien.

- Ancillon, Jean Pierre Frédéric (1767-1837)

Membre de l'Académie de Berlin (1803), précepteur (1808) et maître (1810) du « Kronprinz », futur Frédéric-Guillaume IV, ministre des Affaires extérieures de Prusse (1832).

- Andrié, Jean-Henri, dit A. de Gorgier (1729-1788)

Avant 1765 précepteur de Frédéric-Henri de Prusse, neveu de Frédéric II, anobli par ce dernier (1749).

- Battier, Reinhard (1724-1779)

Éducateur du prince héritier de Saxe-Cobourg-Gotha à Paris (1748-1749), membre de l'Académie de Berlin (1748).

- Béguelin, Nicolas, dit B. de Lichterfelde (1714-1789)

Précepteur du futur Frédéric-Guillaume II (1747-1764), membre (1747), puis directeur de la classe de philosophie de l'Académie de Berlin ; anobli par son ancien élève (1786).

- Bertrand, Elie (1713-1797)

Pasteur à Berne, éducateur des comtes polonais Mniszech dans les années 1760, conseiller du roi Stanislas-Auguste Poniatowski (1765), agrégé à la noblesse polonaise (1768).

- Beylon, Jean-François (1724-1779)

Lecteur de la reine de Suède et chapelain de la Cour (1760), chargé de mission par Gustave III, chevalier de l'Etoile polaire.

- Catt, Henri Alexandre (1725-1795)

Lecteur de Frédéric II et secrétaire de ses commandements (1758-1780), membre de l'Académie de Berlin (1760).

- Crousaz, Jean-Pierre de (1663-1750)

Professeur à l'Académie de Lausanne, gouverneur du prince Frédéric de Hesse-Cassel dans les années 1730, membre associé de l'Académie des sciences de Paris (1735).

- Deyverdun, Jacques-Georges (1734-1789)

Gouverneur des princes de Holstein à Koswig (1761), puis précepteur de Frédéric et de Louis, fils de Frédéric Eugène de Wurtemberg et petit-fils du margrave de BrandebourgSchwedt, de la maison royale de Prusse, ami de Gibbon. 
- Deyverdun, Louise

Gouvernante dans différentes maisons de Saxe et de Poméranie (1760-1765).

- Fornerod

Gouverneur à Moscou chez le général major prince de Galitzine (1785).

- Garcin, Jean-Laurent, dit M. de Cottens (1733-1781)

Précepteur chez Abraham Calkoen, secrétaire de la ville d'Amsterdam, puis séjour académique à Leyde comme gouverneur de deux jeunes Amsteldamois, Gerard et Willem Munter (1763-1766), recherche l'anoblissement.

- Garcin, Jacques (1719-1796)

Gouverneur pour trois grandes maisons de Berlin et de Francfort sur l'oder dans les années 1740, précepteur chez un négociant de Hambourg, pasteur en Hollande et professeur titulaire de la chaire de langue française à Franeker dès 1754.

- Lambert, Jean-Henri (1728-1777)

Précepteur chez le comte P. von Salis à Coire (1748-1756), membre de l'Académie de Berlin (1765).

— Lequin, née de Gélieu, Susanne-Elisabeth (vers 1718-1792)

Partie de Neuchâtel en 1758 suite au décès de son mari, gouvernante en Allemagne, en Livonie et en Russie dans les années 1760, laissa une fortune importante à ses enfants.

- Le Sage, Georges Louis II (1724-1803)

À la fin des années 1740 précepteur, notamment du jeune Friedrich Heinrich Jacobi philosophe allemand, mathématicien et rentier à Genève

- Lhuillier, Simon Antoine Jean (1750-1840)

Précepteur chez les banquiers et négociants genevois Rilliet-Plantamour (1768), gouverneur du prince Czartoryski à la fin des années 1770, membre de l'Académie de Berlin (1789).

- Mallet, Paul Henri (1730-1807)

Précepteur en Haute Lusace chez le comte de Calemberg (1751), chaire de belles-lettres de l'Académie de Copenhague (1752), enseigna la langue et la littérature françaises au prince-royal Christian de Danemark (1752-1760), refusa la place de gouverneur du futur tzar Paul I ${ }^{\text {er }}$, professeur à l'Académie de Genève dès 1761.

- Marat, Jean-Paul (1743-1793)

Précepteur à Bordeaux (1760), maître de français à Edimbourg, médecin des gardes du corps du comte d'Artois, député à la Convention.

- Merian, Jean Bernard (1723-1807)

Précepteur à Amsterdam dans les années 1740, bibliothécaire (1757), directeur de la classe des belles-lettres (1771), secrétaire perpétuel de l'Académie de Berlin (1797).

- Monod, Salomé

Gouvernante chez un chambellan de Prusse vers 1760. 
- Müller, Henriette

Lectrice de la reine-mère de Suède, gouvernante dans plusieurs grandes familles russes dans les années 1770 .

- Muret

Gouverneur au Corps des cadets à Saint-Pétersbourg (1774-1776).

- Prémontval, née Pigeon, Marie-Anne-Victoire

Ep. 1746 André-Pierre Le Guay de P., membre de l'Académie de Berlin (1752), lectrice de la margrave von Schwedt (1752).

- Prevost, Pierre (1751-1839)

Précepteur en Hollande et en France dans les années 1770, membre de l'Académie de Berlin (1780), philosophe.

- Reverdil, Elie Salomon François (1732-1808)

Précepteur des fils du roi Frédéric V de Danemark (1760), secrétaire du cabinet de Christian VII (1766).

- Rousseau, Jean-Jacques (1712-1778)

Précepteur chez les Mably (1740-1741), fit du précepteur une figure centrale de Julie ou La Nouvelle Héloïse et de l'Emile.

- Seedorf, François de Fégely de (1691-1758)

Père jésuite, éducateur et confesseur de l'Electeur palatin Charles-Philippe-Théodore de Sulzbach (1736-1758).

- Sulzer, Johann Georg (1720-1779)

Précepteur à Zurich (1740), puis à Magdebourg (1743), membre (1750) puis directeur de la classe de philosophie de l'Académie de Berlin (1776).

\section{NOTES}

1. Étude d'un réseau de relations savant au XVIII siècle (exemple des correspondants suisses du secrétaire perpétuel de l'Académie de Berlin, 1737-1797, projet financé par le Fonds national suisse de la recherche scientifique, 1997-2001, avec la participation de Cyrille Gigandet, Christian Sester et Thierry Christ. Depuis la parution de l'article, André Bandelier a livré une synthèse intitulée : Des Suisses dans la République des Lettres. Un réseau savant au temps de Frédéric le Grand (Genève, Slatkine, 2007, 216 p.).

2. Jean Sgard (dir.), Dictionnaire des journalistes 1600-1789, Oxford, Voltaire Foundation, 1999, p. 402-406 (article d'Ann Thomson).

3. Dix-huitième siècle, 17, 1985, p. 297-315 (pour le quatrain, p. 305).

4. Documents pour l'histoire du français langue étrangère ou seconde (ci-après Documents), 6, septembre 1990, p. 103-125; 14, décembre 1994, p. 188-210; 18, décembre 1996, p. 63-82. Bilan positif que confirme Jean A. Caravolas, Histoire de la didactique des langues au siècle des Lumières, Les Presses de l'Université de Montréal, 2000, p. 107-169. 
5. André Bandelier, «Un précepteur en Allemagne à la veille de la Révoution, d'après sa correspondance» et "Les métamorphoses d'un maître de français à Londres à la fin du XVIII siècle », dans Documents, 11, juin 1993, p. 37-44;14, décembre 1994, p. 17-24.

6. La qualité de «Suisse » n'est pas limitée à l'origine des correspondants (Suisses des cantons, des pays sujets ou alliés), mais peut être attribuée en fonction de l'action exercée en Suisse (cas d'Antoine Court, restaurateur du protestantisme français). Elle est délibérément reconnue à des savants étrangers qui ont professé ou séjourné temporairement dans ce pays et qui jouent un rôle dans les composantes helvétiques du réseau Formey.

7. Daniel Roche, "Le précepteur, éducateur privilégié et intermédiaire culturel ", dans Les Républicains des lettres. Gens de culture et Lumières au XVIII ${ }^{e}$ siècle, Fayard, 1988, p. 331-349.

8. Johann Heinrich Meister dit Le Maître à Philippe de Rochemont, Bückeburg/Westphalie, 8 mars 1741 (Winterthur, Bibliothek und Briefsammlung Meister, Nachlass von Dekan Johann Heinrich Meister, 6).

9. Werner Hartkopf, Die Berliner Akademie der Wissenschaften. Ihre Mitglieder und Preisträger 1700-1990, Berlin, Akademie Verlag, 1992, 466 p.

10. "L'enseignement du français en Allemagne entre 1648 et 1815 », dans Documents, 18, décembre 1996, p. 69.

11. J'écarte délibérément la volumineuse correspondance de la famille Euler, qui permettrait l'approche de la restauration administrative, intellectuelle et pédagogique de l'Académie impériale de Saint-Pétersbourg, dont dépendait le Corps des cadets. Voir Georges Dulac : «La vie académique à Saint-Pétersbourg vers 1770», dans Académies et sociétés savantes en Europe (1650-1800), Paris, Champion, 2000, p. 221-263

12. Staatsbibliothek zu Berlin, Preussischer Kulturbesitz, Nachlass Formey (ci-après FF), Kasten 24, Lequin née de Gélieu à Formey, 3 novembre 1768 vieux style. Pour des raisons évidentes, les citations respectent la graphie et la ponctuation originales.

13. FF, Kasten 43, Mappe 41, Müller Henriette, 1 lettre, 1775 ; Cracovie, Bibliothèque Jagiellonne, Collection Varnhagen von Ense (ci-après CV), 143, M.V. de Prémontval née Pigeon, 1 lettre, 1757.

14. FF, Kasten 24, Salomé Monod à Formey, Torgau, 12 janvier et 4 février 1760.

15. Lettres d'Elie Luzac à Jean Henri Samuel Formey (1748-1770), éd. par Hans Bots et Jan Schilling, Paris, Honoré Champion, 2001, 415 p.

16. Le Philosophe chrétien, op. cit., I, p. 317 et 321.

17. FF, Kasten 11, I. 15 lettres, 1760-1761, II. 25 lettres, 1763-1765.

18. FF, Kasten 11, Louise Deyverdun à Formey, Torgau, 13 mai 1761.

19. FF, Kasten 11, Louise Deyverdun à Formey, s.l. [Thamenhayn], s.d. [1761].

20. FF, Kasten 11, Louise Deyverdun à Formey, Grauvienckel, 28 juillet 1764.

21. Voir plus loin.

22. FF, Kasten 11, Louise Deyverdun à Formey, Nassenhaide, 10 août 1763 et P.S. octobre 1763.

23. FF, Kasten 11, Louise Deyverdun à Formey, Grauvienckel, 10 février et 10 mars 1765.

24. Voir note 5. H. Duranton, art. cit., p. 298-299, conduit implicitement à la même conclusion à travers les conditions posées par les employeurs.

25. FF, Kasten 17, Garcin (2), 11 lettres, 1763-1765 ; CV, 65, Garcin (Leiden), 3 lettres, 1764-1765. Pour les «Hollandais» du corpus, notamment Jean-Laurent et Jacques Garcin, j'ai bénéficié de l'appui précieux de Marie-Christine Kok Escalle et de Kees van Strien, auxquels va toute ma gratitude.

26. Informations tirées des archives de la famille Calkoen (Algemen Rijksarchief den Haag, index 1.10.16.01) et de Album Studiosorum Lugdun., col. 1079, 14 septembre 1763.

27. FF, Kasten 17, Garcin (2), Jean-Laurent Garcin à Formey, s.l. [Amsterdam], 1763.

28. Jean Sgard (dir.), op. cit., méconnaît l'activité journalistique de Jean-Laurent Garcin (l'article Laurent Garcin est consacré à son père, un des collaborateurs assidus du Mercure suisse et du Journal helvétique de Neuchâtel, p. 434-435) et le Dictionnaire des journaux, Oxford, Voltaire 
Foundation, 1991, ne cite pas non plus la continuation hollandaise du Journal étranger (p. 674-675).

29. André Bandelier, «Formey, Jean Henri Samuel (Berlin, 1711 - Berlin, 1797)», dans Dictionnaire de Jean-Jacques Rousseau, sous la dir. de Raymond Trousson et Frédéric S. Eigeldinger, Paris, Honoré Champion, 1996, p. 346-348. Je prépare un article sur l'impression de l'Emile chrétien, de Formey, pour le Bulletin de l'Association Jean Jacques Rousseau (Bibliothèque publique et universitaire de Neuchâtel). La correspondance porte par ailleurs sur la publication à Leyde des Discours philosophiques de Maxime de Tyr, traduits du grec par Formey, et des Principes de morale, déduits de l'usage des facultés de l'entendement humain, du même auteur.

30. V.d.Aa, Biographisch woordenboek der Nederlanden, Haarlem, JJ van Brederode, 1869, 7e deel, p. 41.

31. FF, Kasten 17, Garcin (1), Jacques Garcin à Formey, Franeker, 27 septembre 1763.

32. FF, Kasten 44, Bl. 831-832, 866-869, 2 lettres, 1785 ; FF, Kasten 16, 1 lettre, 1786.

33. Les citations sont tirées des deux lettres de 1785 : FF, Kasten 44, Fornerod à Formey, Moscou, $8 / 19$ mai et $8 / 19$ octobre.

34. FF, Kasten 26, 12 lettres, 1751-1758 ; Staatsbibliothek zu Berlin, Preussischer Kulturbesitz, Sammlung Darmstaedter (ci-après SD), 2 f 1760 (1), 7 lettres, 1751, 1757, s.d. ; CV, 112, 24 lettres, 1751-1763.

35. FF, Kasten 26, Mallet à Formey, s.l., s.d. [de Lusace, septembre ou octobre 1751].

36. Avec le recul, il jugera son attitude présomptueuse : voir dernière lettre du dossier (1763) où il répond à Formey sur la demande du comte de Calemberg qui veut placer son fils à Genève.

37. La citation, comme les précédentes, est tirée de SD, 2 f 1760 (1), Mallet à Formey, s.l., s.d. [de Lusace, décembre 1751].

38. The complete works of Voltaire, Voltaire's Correspondence, ne garde pas trace de la sollicitation de Formey à ma connaissance. Voir pour le jugement de l'«Ode aux Prussiens», de Mallet, parue dans l'Abeille du Parnasse : vol. 96, D 4452, Voltaire à Formey, Potsdam, 30 avril [1751].

39. Claude Lauriol, « Angliviel de La Beaumelle, Laurent (1726-1773) », dans Jean Sgard (dir.), Dictionnaire des journalistes, op. cit., p. 12-17.

40. $\mathrm{CV}, 112$, Mallet à Formey, Copenhague, mai 1754.

41. CV, 112, Mallet à Formey, Copenhague, 24 janvier 1756.

42. $\mathrm{CV}, 112$, Mallet à Formey, Copenhague, 8 novembre 1757.

43. Cyrille Gigandet, «...Arracher quelque portion de ces odieuses bariéres qui nous divisent...» : analyse des préfaces aux traductions «nordiques» du Genevois Paul-Henri Mallet», dans La vie intellectuelle aux refuges protestants. II. Huguenots traducteurs, Actes de la Table ronde de Dublin, juillet 1999, J. Häseler et A. McKenna (dir.), Paris, Champion, 2002, p. 153-174.

44. Le père est sans doute Henri Frédéric comte de Frise (1681-1739), général saxon, une des personnalités les plus influentes à la Cour de Dresde, et l'héritier son fils Auguste Henri, né en 1726, mort maréchal de camp français à Chambord en 1755 : Allgemeine deutsche Biographie, Leipzig, Duncker \& Humblot, vol. VIII, 1878, p. 88 ; Neue deutsche Biographie, Berlin, Duncker \& Humblot, vol. V, 1961, p. 611-612.

45. Joël Cornette, "Petit traité d'éducation des princes », dans L'Histoire, 262, février 2002, p. 44-45.

46. SD, 2 a 1733 (1), Jean-Pierre de Crousaz au général de Crousaz en Saxe, Lausanne, 7 juin 1735.

47. Henri Besse, " Propositions pour une typologie des méthodes de langues ", dans Documents, 26, juin 2001, p. 158-159.

48. Traité de l'éducation, op. cit., « Des langues », p. 250 sq.

49. Voir note 46 : de «la feuille que vous lui feres lire» (Crousaz); d'une autre main : «Lettres sur l'Education par Mr. Le Professeur J.P. de Crousaz addressées a Mr le General de Crousaz en Saxe». 50. Konrad Schröder dresse un portrait de Béguelin dans « Französichunterricht in Berlin im 18. Jahrhundert », Documents, 14, décembre 1994, p. 202-203. 
51. FF, Kasten 4, 44 lettres, 1748-1788 ; SD, F 1 f1764 (1), 17 lettres, 1748-1787 ; CV, 27, 14 lettres, 1748-1787.

52. FF, Kasten 11, 24 lettres, 1761-1766 ; CV, 52, 6 lettres, 1761-1767.

53. Von Feilitsch réussit à le tenir éloigné de son aîné Frédéric (1754-1816), le premier roi du Wurtemberg moderne; il se console avec Louis (1756-1817), futur gouverneur de Varsovie et irascible époux de la princesse Maria Anna Czartoryska. Les deux frères, de confession réformée contrairement à leur père, firent un séjour d'études de trois ans à Lausanne au début des années 1770. Pour les enfants de Frédéric Eugène: Das Haus Württemberg ein biographisches Lexikon, Stuttgart, Kohlhammer, 1997, p. 273 sq. (voir 6.0.3. Friedrich II./I. et 6.1.1. Ludwig, «Louis»).

54. Les quatre citations sont tirées successivement de: FF, Kasten 11, Jacques-Georges Deyverdun à Formey, s.l., 1er janvier 1763 ; id., Schwedt, 10 juin 1763 ; id., s.l., 11 mai 1763 ; CV, 52, Londres, 1er mars 1766.

55. FF, Kasten 4, 1 lettre, 1763 ; CV, 30, 6 lettres, 1763-1777.

56. CV, 30, Beylon à Formey, Stockholm, 29 août 1777.

\section{RÉSUMÉS}

Les Lumières sont réputées siècle d'or de la correspondance. Les études récentes s'attachent aux réseaux qu'on peut induire de fonds épistolaires exceptionnels. Conservée à Berlin et à Cracovie, la correspondance de Samuel Formey appartient à ceux-ci. Au centre d'un échange important de précepteurs et gouvernantes qui couvre toute la seconde moitié du XVIII ${ }^{\mathrm{e}}$ siècle, elle semblait particulièrement apte à approcher l'enseignement privé du français, langue internationale. En fait, à l'aune de l'échantillon suisse (quelques dizaines de précepteurs, peu de gouvernantes), elle offre rarement les à-peu-près des pratiques d'avant la professionnalisation. Elle permet de cerner la condition de ces intermédiaires culturels clés d'une société, de repérer aussi les manifestations d'une Europe francophone, nordique et orientale.

The Age of Enlightenment is considered a golden century for the correspondence. The recent studies become attached to the networks which we can infer of exceptional epistolary funds. Preserved in Berlin and in Cracow, the correspondence of Samuel Formey belongs to these. In the center of an important exchange of private tutors and governesses which covers all second half of the XVIIIth century, it seemed particularly suitable of approaching the private teaching of French as an international tongue. In fact, if we consider the Swiss sample (several dozens of private tutors, a few governesses), it rarely offers an approximation of the practices from before the professionalization. It allows to encircle the condition of these key cultural intermediaries of a society, to locate also the demonstrations of a French-speaking Europe, scandinavian and oriental Europe included.

\section{INDEX}

Mots-clés : europe francophone, gouvernantes, précepteurs, Samuel Formey, Suisse, XVIIIe siècle

Keywords : governesses, private tutors, Samuel Formey, Switzerland, XVIII century 
AUTEUR

ANDRÉ BANDELIER

Université de Neuchâtel 